\title{
Long-Term Effects of Apixaban Confirmed in the Open-Label Extension of AVERROES Trial
}

\author{
Tze-Fan Chao ${ }^{1,2}$ Tatjana S. Potpara ${ }^{3,4}$ \\ ${ }^{1}$ Division of Cardiology, Department of Medicine, Taipei Veterans \\ General Hospital, Taipei, Taiwan \\ 2 Institute of Clinical Medicine and Cardiovascular Research Center, \\ National Yang-Ming University, Taipei, Taiwan \\ ${ }^{3}$ School of Medicine, Belgrade University, Belgrade, Serbia \\ ${ }^{4}$ Cardiology Clinic, Clinical Centre of Serbia, Belgrade, Serbia \\ Thromb Haemost 2021;121:405-407.
}

The efficacy and safety of non-vitamin K antagonist (non-VKA) oral anticoagulants (NOACs) compared with warfarin have been shown in four pivotal randomized trials. ${ }^{1-4}$ Thereafter, NOACs have been increasingly prescribed for stroke prevention in the daily practice, thus improving clinical outcome for atrial fibrillation (AF) patients. ${ }^{5}$ The use of apixaban for AF-related stroke prevention has been studied in two randomized trials, the AVERROES (Apixaban Versus ASA to Prevent Stroke in AF Patients Who Have Failed or Are Unsuitable for Vitamin K Antagonist Treatment) and ARISTOTLE (Apixaban for Reduction in Stroke and Other Thromboembolic Events in Atrial Fibrillation). ${ }^{3,6}$ The AVERROES trial randomized AF patients considered unsuitable for VKA to receive aspirin or apixaban, and showed superiority of apixaban over aspirin for stroke prevention with comparable risks of intracranial hemorrhage and major bleeding. ${ }^{6}$ Hence, apixaban is the only NOAC which has been compared with aspirin in a randomized trial for stroke prevention in AF. However, the median follow-up in the AVERROES trial was only 1 year, and data regarding the longterm outcome of apixaban versus aspirin are unknown.

In this issue of Thrombosis and Haemostasis, Benz and colleagues reported outcomes among 3,275 patients on apixaban during the open-label extension of the AVERROES trial. ${ }^{7}$ After a 3-year median follow-up, the rate of stroke/systemic embolism (SE) was 1.0\%/year, and the risk of major bleeding was $1.2 \%$ year. These event rates were similar to those observed among patients receiving apixaban during the double-blinded phase of AVERROES. The findings of the present study confirm the long-term effectiveness and safety of apixaban for stroke prevention in AF patients.

Previous studies have reported the risk of stroke/SE and major bleeding among patients randomized to a NOAC during and after the respective double-blinded phase..$^{8-11}$ These data and those reported by Benz et al are summarized in - Fig. 1. A higher risk of stroke/SE during the open-label phase was
Address for correspondence Tatjana Potpara, MD, PhD, School of Medicine, Belgrade University, dr Subotica 8, Cardiology Clinic, Clinical Centre of Serbia, Visegradska 26, 11000 Belgrade, Serbia (e-mail: tatjana.potpara@med.bg.ac.rs).

observed among patients randomized to NOACs and then transitioned to an open-label VKA in the ROCKET-AF (6.42\%/ year) and ARISTOTLE (4.02\%/year) trials. ${ }^{8,11}$ Of note, the event rate among nonanticoagulated $\mathrm{AF}$ patients with a mean CHADS $_{2}$ score of 3.5 was 5.9\%/year for a score of 3 and $8.5 \% /$ year for a score of 4 in the study by Gage et al. ${ }^{12}$

Learning from the ROCKET-AF and ARISTOTLE trials, a delicate transition plan was set up in the ENGAGE-AF TIMI 48. ${ }^{10}$ Owing to the aggressive monitoring and titration of VKA therapy, the median time to first therapeutic international normalized ratio (INR) $\geq 2$ on open-label VKA was shorter in the ENGAGE AF-TIMI 48 (9 days) compared with ROCKET-AF (13 days). ${ }^{8,10}$ Obviously, a 2-day "bridging" period with apixaban was too short to achieve a therapeutic INR among patients who transited from apixaban to openlabel VKA in the ARISTOTLE trial. ${ }^{11}$ By 30 days after the end of the trial, at least one therapeutic INR was achieved in around $99 \%$ of patients randomized to edoxaban and transitioned to open-label VKA in ENGAGE AF-TIMI 48, compared with only $52 \%$ in the ROCKET-AF trial. The risk of stroke/SE in patients randomized to edoxaban during the transition phase was similar to that in the double-blinded phase in ENGAGE-AF TIMI 48 (1.89\%/year vs. $1.57 \%$ with high-dose edoxaban regimen; $1.85 \% /$ year versus $2.04 \% / y e a r$ with low-dose edoxaban regimen)..$^{10}$ These observations highlighted the importance of a comprehensive transition program when patients were shifted from a NOAC to VKA.

The RELY-ABLE study ${ }^{9}$ (an open-label extension of the RE-LY trial) and the present report by Benz et al represent the body of evidence regarding long-term outcomes of NOAC use during open-label extension of landmark trials on stroke prevention in AF. The long-term risk of major bleeding with apixaban was consistently low (1.2\%/year) even among patients who were considered unsuitable for VKA at the AVERROES trial entry, thus suggesting that NOACs could provide an easier and better published online January 20, 2021 (c) 2021. Thieme. All rights reserved. Georg Thieme Verlag KG,

Rüdigerstraße 14,

70469 Stuttgart, Germany
DOI https://doi.org/ 10.1055/a-1367-0184. ISSN $0340-6245$. 

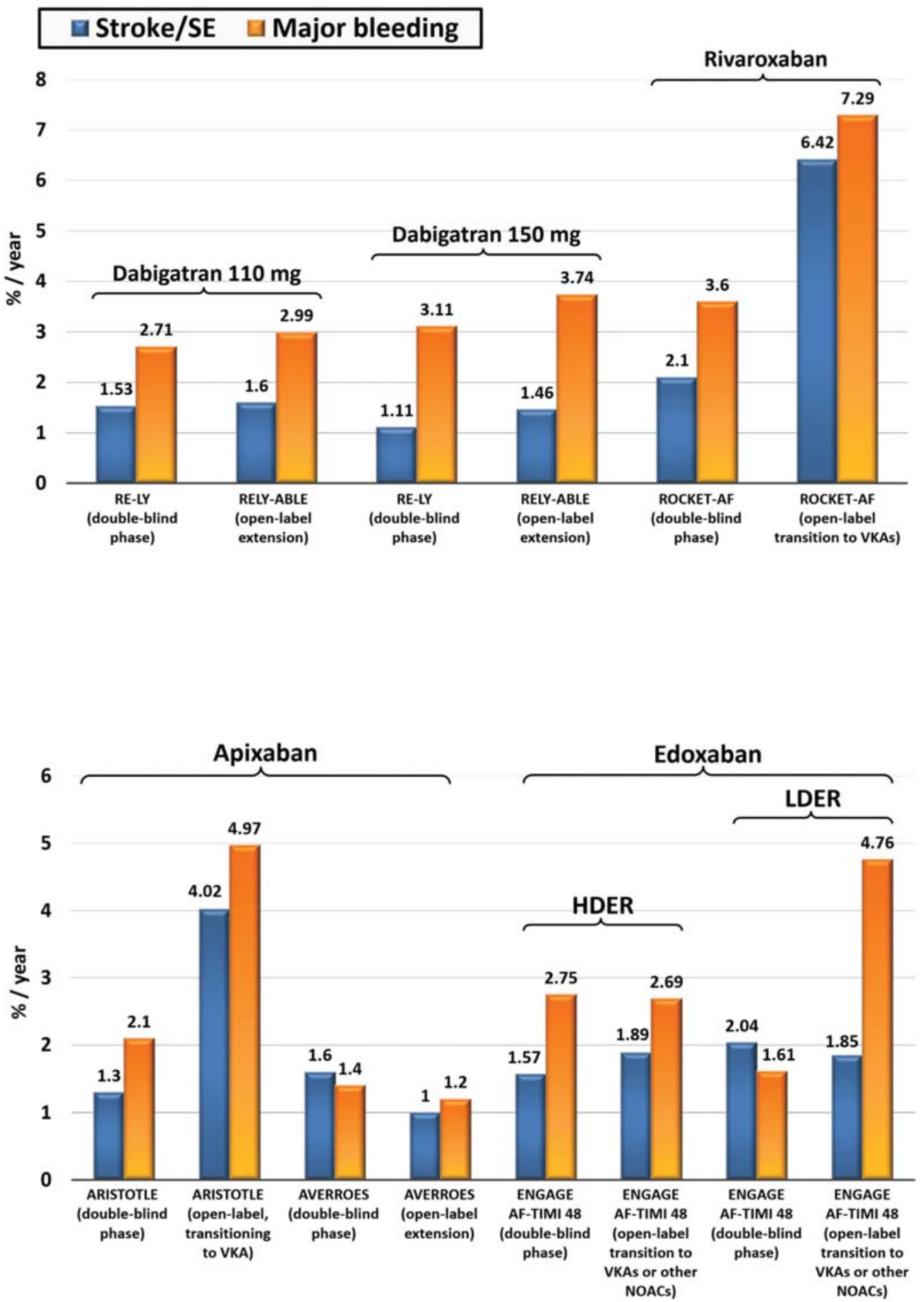

Fig. 1 Risks of stroke/SE and major bleeding of patients who had been randomized to an NOAC during and after the end of double-blind phase, and those reported in the open-label extension period of RE-LY (RELY-ABLE) and AVERROES trials. Data used in the figure were adopted from RELY, ${ }^{1}$ ROCKET-AF, ${ }^{2}$ AVERROES, ${ }^{6}$ ARISTOTLE, ${ }^{3}$ ENGAGE-AF TIMI 48 trials, ${ }^{4}$ RELY-ABLE study, ${ }^{9}$ and the studies by Mahaffey et al, ${ }^{8}$ Ruff et al, ${ }^{10} \mathrm{Granger}$ et al, ${ }^{11}$ and Benz et al. ${ }^{7}$ HDER, high-dose edoxaban regimen; LDER, low-dose edoxaban regimen; NOAC, non-vitamin $\mathrm{K}$ antagonist oral anticoagulant; SE, systemic embolism; VKA, vitamin K antagonist.

way than VKA to prevent stroke in AF patients. Actually, more and more patients who would be considered unsuitable for oral anticoagulant therapy with VKA only a few years ago now seem to do relatively well on NOACs, e.g., the extremely elderly and patients with prior history of intracranial hemorrhage. ${ }^{13,14}$ The data from randomized trials seem to be well augmented and supported by a wealth of real-world data despite the higher risk profiles of patients in observational nontrial cohorts. ${ }^{15,16}$

When interpreting results of the open-label extension studies, we should realize that these patients were followed up regularly (e.g., 1, 6, and 12 months after entry into the openlabel extension of AVERROES, and every 6 months thereafter), 
and therefore, each participant was well managed. As emphasized in the 2020 European Society of Cardiology AF Guidelines, ${ }^{17}$ careful evaluation and characterization of the patient, ${ }^{18}$ as well as a regular follow-up, risk reassessment (even in those initially considered as low risk, given the dynamic nature of stroke and bleeding risks), ${ }^{19,20}$ and management of modifiable bleeding risk factors are crucial for the care of anticoagulated AF patients. Indeed, the well-structured, easyto-use ABC holistic pathway (A: Avoid stroke; B: Better symptom management; $C$ : Cardiovascular and other comorbidity risk reduction), should be implemented into daily practice for the management of all AF patients. ${ }^{21,22}$

\section{Conflict of Interest}

None declared.

\section{References}

1 Connolly SJ, Ezekowitz MD, Yusuf S, et al; RE-LY Steering Committee and Investigators. Dabigatran versus warfarin in patients with atrial fibrillation. N Engl J Med 2009;361(12):1139-1151

2 Patel MR, Mahaffey KW, Garg J, et al; ROCKET AF Investigators. Rivaroxaban versus warfarin in nonvalvular atrial fibrillation. N Engl J Med 2011;365(10):883-891

3 Granger CB, Alexander JH, McMurray JJ, et al; ARISTOTLE Committees and Investigators. Apixaban versus warfarin in patients with atrial fibrillation. N Engl J Med 2011;365(11):981-992

4 Giugliano RP, Ruff CT, Braunwald E, et al; ENGAGE AF-TIMI 48 Investigators. Edoxaban versus warfarin in patients with atrial fibrillation. N Engl J Med 2013;369(22):2093-2104

5 Chao TF, Chiang CE, Lin YJ, et al. Evolving changes of the use of oral anticoagulants and outcomes in patients with newly diagnosed atrial fibrillation in Taiwan. Circulation 2018;138(14):1485-1487

6 Connolly SJ, Eikelboom J, Joyner C, et al; AVERROES Steering Committee and Investigators. Apixaban in patients with atrial fibrillation. N Engl J Med 2011;364(09):806-817

7 Benz AP, Eikelboom JW, Yusuf S, et al. Long-term treatment with apixaban in patients with atrial fibrillation: outcomes during the open-label extension following AVERROES. Thromb Haemost 2021;121(04):518-528

8 Mahaffey KW, Hellkamp AS, Patel MR, et al. End of study transition from study drug to open-label vitamin K antagonist therapy: the ROCKET AF experience. Circ Cardiovasc Qual Outcomes 2013; 6(04):470-478

9 Connolly SJ, Wallentin L, Ezekowitz MD, et al. The long-term multicenter observational study of dabigatran treatment in patients with atrial fibrillation (RELY-ABLE) study. Circulation 2013;128(03):237-243

10 Ruff CT, Giugliano RP, Braunwald E, et al. Transition of patients from blinded study drug to open-label anticoagulation: the ENGAGE AF-TIMI 48 trial. J Am Coll Cardiol 2014;64(06):576-584
11 Granger CB, Lopes RD, Hanna M, et al. Clinical events after transitioning from apixaban versus warfarin to warfarin at the end of the Apixaban for Reduction in Stroke and Other Thromboembolic Events in Atrial Fibrillation (ARISTOTLE) trial. Am Heart J 2015;169(01):25-30

12 Gage BF, Waterman AD, Shannon W, Boechler M, Rich MW, Radford MJ. Validation of clinical classification schemes for predicting stroke: results from the National Registry of Atrial Fibrillation. JAMA 2001;285(22):2864-2870

13 Chao TF, Liu CJ, Lin YJ, et al. Oral anticoagulation in very elderly patients with atrial fibrillation: a nationwide cohort study. Circulation 2018;138(01):37-47

14 Tsai CT, Liao JN, Chiang CE, et al. Association of ischemic stroke, major bleeding, and other adverse events with warfarin use vs non-vitamin $\mathrm{K}$ antagonist oral anticoagulant use in patients with atrial fibrillation with a history of intracranial hemorrhage. JAMA Netw Open 2020;3(06):e206424

15 de Vries TAC, Hirsh J, Xu K, et al. Apixaban for stroke prevention in atrial fibrillation: why are event rates higher in clinical practice than in randomized trials?-a systematic review Thromb Haemost 2020;120(09):1323-1329

16 Hohmann C, Hohnloser SH, Jacob J, Walker J, Baldus S, Pfister R. Non-vitamin $\mathrm{K}$ oral anticoagulants in comparison to phenprocoumon in geriatric and non-geriatric patients with non-valvular atrial fibrillation. Thromb Haemost 2019;119(06): 971-980

17 Hindricks G, Potpara T, Dagres N, et al; ESC Scientific Document Group. 2020 ESC Guidelines for the diagnosis and management of atrial fibrillation developed in collaboration with the European Association of Cardio-Thoracic Surgery (EACTS). Eur Heart J 202010.1093/eurheartj/ehaa612

18 Potpara TS, Lip GYH, Blomstrom-Lundqvist C, et al. The 4S-AF scheme (stroke risk; symptoms; severity of burden; substrate): a novel approach to in-depth characterization (rather than classification) of atrial fibrillation. Thromb Haemost 2020

19 Choi SY, Kim MHH, Lee KM, et al. Age-dependent anticoagulant therapy for atrial fibrillation patients with intermediate risk of ischemic stroke: a nationwide population-based study. Thromb Haemost 2020. Doi: 10.1055/a-1336-0476

20 Chao TF, Liao JN, Tuan TC, et al. Incident co-morbidities in patients with atrial fibrillation initially with a CHA2DS2-VASc score of 0 (males) or 1 (females): implications for reassessment of stroke risk in initially 'low-risk' patients. Thromb Haemost 2019;119 (07):1162-1170

21 Yoon M, Yang PS, Jang E, et al. Improved population-based clinical outcomes of patients with atrial fibrillation by compliance with the simple ABC (Atrial Fibrillation Better Care) pathway for integrated care management: a nationwide cohort study. Thromb Haemost 2019;119(10):1695-1703

22 Guo Y, Guo J, Shi X, et al; mAF-App II Trial investigators. Mobile health technology-supported atrial fibrillation screening and integrated care: a report from the mAFA-II trial Long-term Extension Cohort. Eur J Intern Med 2020;82:105-111 